\title{
Vomiting-induced costal cartilage fracture: a case report
}

\author{
Eleni Drakonaki ${ }^{1}$, Ioannis Karageorgiou², Stamatios Kokkinakis ${ }^{2}$, Neofytos Maliotis ${ }^{2}$, Rania \\ Spyridaki $^{3}$, Emmanouil K Symvoulakis ${ }^{2}$
}

\author{
${ }^{1}$ Department of Anatomy, European University of Cyprus Medical School, Nicosia, Cyprus, ${ }^{2}$ Clinic of Social and \\ Family Medicine, School of Medicine, University of Crete, Heraklion, Greece, ${ }^{3}$ Private Hospital Creta Interclinic, \\ Heraklion, Greece
}

\begin{abstract}
The use of ultrasonography as a first line imaging test in cases of possible costal cartilage fracture can be pivotal. In this case report, we present the case of a patient with a suspected atraumatic vomiting-induced costal cartilage fracture. The costal cartilage fracture was non-displaced and incomplete, thus not visible in a Computed Tomography scan. When Ultrasound imaging was employed at the area of tenderness, soft tissue edema and hematoma around the cartilage were visualized. High level of suspicion for a cartilage fracture in this case revealed a subtle osseous injury.

Keywords: costal cartilage; fractures, cartilage; vomiting; ultrasonography
\end{abstract}

\section{Introduction}

Thoracic cage injuries present a wide range of potential diagnoses. Costal cartilage (CC) injuries pose a diagnostic challenge, often being missed in the initial assessment involving a chest radiograph. Costal cartilage fractures should be considered, especially in cases of blunt trauma involving contact sports athletes or high-energy trauma, such as motor vehicle accidents [1]. These fractures are often underdiagnosed, while atraumatic cases have rarely been reported in the literature [2]. In this article, a case of vomiting-induced costal cartilage fracture is presented.

\section{Case report}

A 53-year-old woman presented at a primary care service institution due to significant pain at the left hemithorax. The pain was more intense during deep breathing

Received 17.06.2020 Accepted 25.09.2020

Med Ultrason

2022, Vol. 24, No 1, 117-119

Corresponding author: Emmanouil K Symvoulakis

Assistant Professor of Primary Health Care

Clinic of Social and Family Medicine,

School of Medicine, University of Crete, Greece

Voutes, 71003, Heraklion, Greece

Phone: +302810394621

Email: symvouman@yahoo.com and coughing. The patient reported that the pain had appeared suddenly six days earlier when standing against a hard surface at the toilet while vomiting. She also reported that she had several episodes of vomiting in the last week, due to an episode of gastroenteritis.

Clinical examination revealed no significant abnormality. In palpation, there was an area of significant tenderness over the left costal margin near the left sternocostal synchondrosis. An ultrasonography (US) of the area of pain was ordered, which revealed the findings described in figure 1.

A CT scan was ordered to rule out a rib fracture. CT images of the thorax identified no rib fracture. At axial CT images there was a tiny low-density area at the seventh costal cartilage and swelling of the adjacent soft tissue. As the CT was non-diagnostic, further investigation with MRI was performed (fig 2). Interestingly, the MRI revealed a hyperintense linear area at the superior part of the seventh costal cartilage that corresponded to the tiny low density area identified in the CT. The diagnosis of a vertical incomplete fracture of the seventh costal cartilage with associated soft tissue edema secondary to injury or significant vomiting was made.

Analgesics and rest were recommended. At follow up four weeks later, U/S showed that the soft tissue edema/ hematoma had partially resolved and the patient was feeling significantly better. 


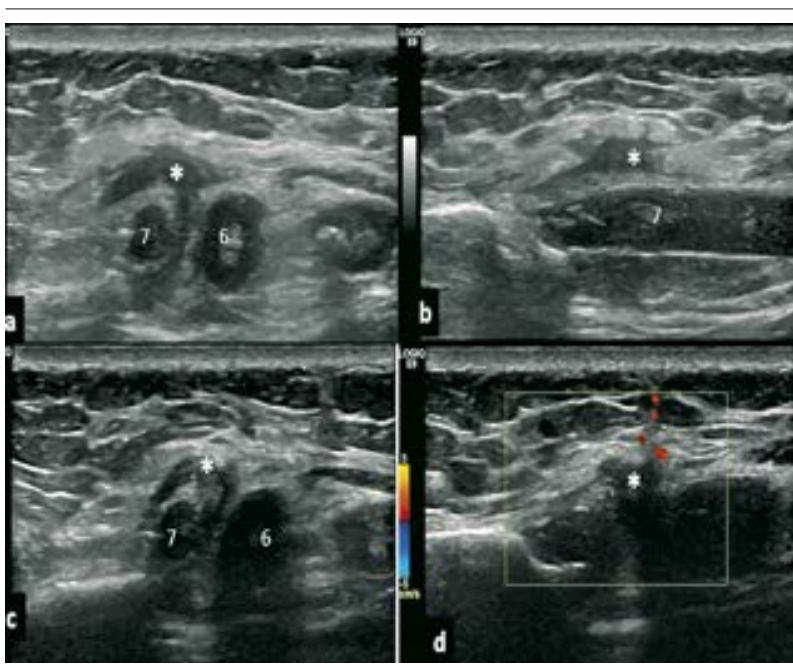

Fig 1. Ultrasound images over the painful area at the LT costal margin. Axial (a) and longitudinal (b) B-mode US images showing the seventh and sixth left costal cartilage seven days after injury. There is an irregular hypoechoic area (asterisk) surrounding the seventh costal cartilage and the intercostal space in keeping with hematoma. The subcutaneous tissue overlying the hematoma is hyperechoic, which is probably secondary to inflammation or hemorrhagic infiltration from the hematoma. No fracture line is identified. Axial (c) and Longitudinal (d) B-mode US images showing the seventh and sixth left costal cartilage 30 days after injury. The hypoechoic area overlying the costal cartilage has diminished in size, suggesting that the hematoma has partially resolved. Doppler imaging (d) shows limited vascularity at the subcutaneous tissue overlying the area of injury and hematoma, suggesting an ongoing healing procedure.

\section{Discussion}

After a brief literature review, we found that the most well described mechanism of injury that approaches vomiting is vigorous coughing. According to a recent study, costal cartilage fracture due to violent coughing was reported in two cases [2]. In our case, recurrent vomiting induced a costal cartilage fracture. We speculate that this may be another potential cause of CC injury that has not been previously reported.

The natural history of costal cartilage fracture remains unclear. Observing the cartilage and soft-tissue abnormalities of the anterior chest wall after injury is often difficult with the radiographs due to their insensitivity. However, eligible techniques that are more sensitive in revealing these types of injuries include CT, MRI and US [3-5].

Treatment depends on the location and severity of the fracture. Management in cases of single non-displaced $\mathrm{CC}$ fractures is primarily conservative. As far as patients with more complex trauma are concerned, operative management may be beneficial in specific patient subgroups although this is not certain [6].
Even though the usefulness of CT in diagnosing costal cartilage fractures is well established [7] the same is not true for MRI. There are some reports comparing findings between diagnostic modalities [8], but are of limited value, due to the small number of cases they examine. The same is true for the role of US [9]. What the authors seem to agree on is that costal cartilage fractures are underdiagnosed [7] due to the lack of a reliable and readily accessible imaging technique.

Our case was special, for two main reasons. Firstly, patient history and presentation was atypical. Costal cartilage fractures occur either as sports injuries in contact sports [4] or in case of high-energy blunt force trauma such as motor vehicle accidents [3]. As a result, costal cartilage fractures tend to occur in middle-aged males [7]. Secondly, because the fracture was not displaced and incomplete, a CT scan diagnosis was difficult to establish. In our case, MRI was more sensitive in detecting the high signal intensity fracture line.

Finally, we would like to use this case as an opportunity to point out that US can be used as the first line imaging test guided by the area of tenderness and pain and showing soft tissue edema and hematoma around the

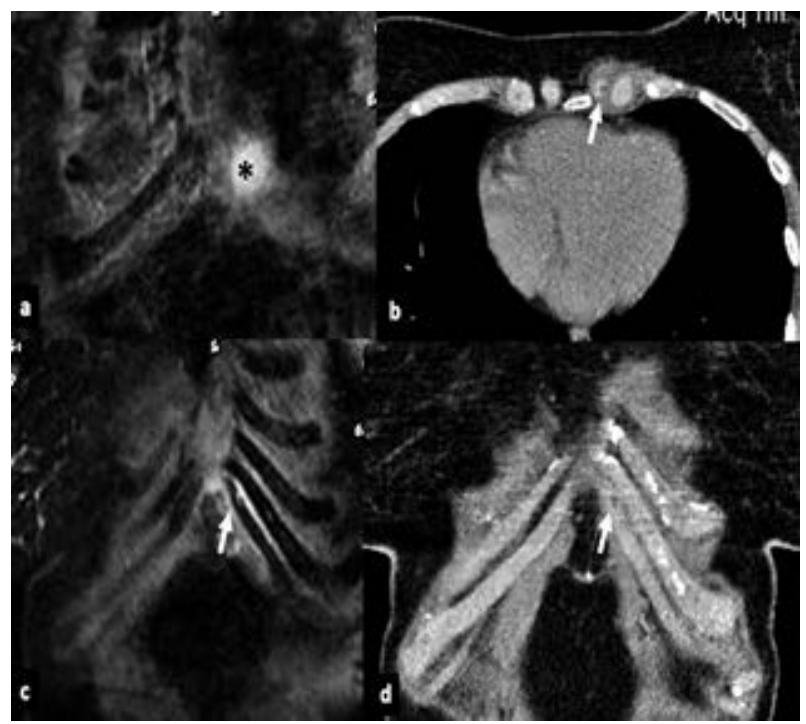

Fig 2. Coronal (a), (c) fat-suppressed T2-weighted images at the painful left costosternal area reveals a hyperintense linear area at the superior part of the seventh costal cartilage that corresponds to a vertical incomplete fracture (arrow at c), accompanied by hyperintense soft tissue edema/hematoma at the adjacent intercostal spaces (c) and the superficial soft tissues (a). Axial (b) and corresponding coronal (d) CT images of the thorax at the area of injury reveals a tiny low density area at the seventh costal cartilage (arrows at b and d) that corresponds to the incomplete costal cartilage fracture. Significant soft-tissue edema/hematoma superficial and deep to the sixth and seventh costal cartilage due to contusion injury is also present. 
cartilage. If the US is positive, CT or MRI can be considered, depending on patient history, physical findings, and clinical suspicion.

In conclusion, to the best of our knowledge, no vomiting-induced costal cartilage fracture has previously been reported in the literature. A high level of suspicion is required to make a correct diagnosis in atraumatic cases. In non-displaced fractures, MRI seems to be superior compared to chest $\mathrm{CT}$ and more studies regarding its value are required. Ultrasound examination of the affected area can either lead to a diagnosis in complicated cases or imply that further imaging is needed, as in our case.

\section{References}

1. Kani KK, Mulcahy H, Porrino JA, Chew FS. Thoracic Cage Injuries. Eur J Radiol 2019;110:225-232.

2. Daniels SP, Kazam JJ, Yao KV, Xu HS, Green DB. Cough-induced costal cartilage fracture. Clin Imaging 2019;55:161-164.

3. Nummela MT, Bensch FV, Pyhältö TT, Koskinen SK. Incidence and Imaging Findings of Costal Cartilage Fractures in Patients with Blunt Chest Trauma: A Retrospective Re- view of 1461 Consecutive Whole-Body CT Examinations for Trauma. Radiology 2018;286:696-704.

4. McAdams TR, Deimel JF, Ferguson J, Beamer BS, Beaulieu CF. Chondral rib fractures in professional American football: two cases and current practice patterns among NFL team physicians. Orthop J Sports Med 2016;4:2325967115627623.

5. Subhas N, Kline MJ, Moskal MJ, White LM, Recht MP. MRI evaluation of costal cartilage injuries. AJR Am J Roentgenol 2008;191:129-132.

6. Yuan SM. Isolated costal cartilage fractures: the radiographically overlooked injuries. Folia Morphol (Warsz) 2017;76:139-142.

7. Malghem J, Vande Berg B, Lecouvet F, Maldague B. Costal Cartilage Fractures as Revealed on CT and Sonography. AJR Am J Roentgenol 2001;176:429-432.

8. Tomas X, Facenda C, Vaz N, et al. Thoracic wall trauma-misdiagnosed lesions on radiographs and usefulness of ultrasound, multidetector computed tomography and magnetic resonance imaging. Quant Imaging Med Surg 2017;7:384-397.

9. Griffith JF, Rainer TH, Ching AS, Law KL, Cocks RA, Metreweli C. Sonography Compared With Radiography in Revealing Acute Rib Fracture. AJR Am J Roentgenol 1999;173:1603-1609. 